\title{
Concomitant Acute Hepatic Failure and Renal Failure Induced by Intravenous Amiodarone: A Case Report and Literature Review
}

\author{
Mujtaba Mohamed ${ }^{\mathrm{a}, \mathrm{b}}$, Alsadiq Al-Hillan ${ }^{\mathrm{a}}$, Marcus Flores ${ }^{\mathrm{a}}$, Christian Kaunzinger ${ }^{\mathrm{a}}$, \\ Arman Mushtaq ${ }^{\mathrm{a}}$, Arif Asif ${ }^{\mathrm{a}}$, Mohammad Hossain ${ }^{\mathrm{a}}$
}

\begin{abstract}
Hepatotoxicity caused by chronic oral amiodarone is well documented with around $15-20 \%$ incidence rate. However, acute liver failure due to intravenous (IV) amiodarone is rare clinical presentation with $3 \%$ incidence rate. Incidence of concomitant renal failure is even rarer. There is no full explanation for the underlying mechanism. Herein, we are presenting a rare case of concomitant acute hepatic failure and acute-on-chronic renal injury induced by use of IV amiodarone. A 67-year-old man with past medical history of coronary artery disease $\mathrm{s} / \mathrm{p}$ coronary artery bypass graft $(\mathrm{CABG})$, history of alcoholism, and chronic kidney disease stage 3 presented with chest pain for 1 week. In the emergency department (ED), he was found to have atrial flutter. Due to unresponsiveness to IV $\beta$-blocker and diltiazem, the patient was loaded with IV amiodarone and continued IV amiodarone drip. His liver function tests (LFTs) and renal functions at the time of administration of IV amiodarone were aspartate transaminase (AST) $176(10-42 \mathrm{IU} / \mathrm{L})$ and alanine transaminase (ALT) 208 (10 - 60 IU/L), international normalized ratio (INR) 1.39 (reference value 2 3), blood urea nitrogen (BUN) 37 (5-25 mg/dL), and creatinine 1.85. Sixteen hours later patient developed acute hepatic failure with AST 4,250 (reference value 10 - 42 IU/L), ALT 2,422 (10 - 60 IU/L), INR 2.28 , and acute renal failure with creatinine of $3.2 \mathrm{mg} / \mathrm{dL}(0.44-1.0$ $\mathrm{mg} / \mathrm{dL})$, and BUN of $44 \mathrm{mg} / \mathrm{d}(5-25 \mathrm{mg} / \mathrm{dL})$. Patient was intubated due to acute hepatic encephalopathy and sent to intensive care unit (ICU). IV amiodarone was stopped immediately. All workup for other causes of acute hepatic failure came back negative. He was started on IV N-acetylcysteine and required hemodialysis for acute-on-chronic renal failure. LFTs peaked $72 \mathrm{~h}$ after discontinuation of amiodarone. Kidney functions started to improve 5 days after discontinuation of amiodarone and patient came off hemodialysis. Acute hepatic failure as result of IV amiodarone is a rare presentation; however, it has a high mortality. Risk factors include low ejection fraction, hepatic con-
\end{abstract}

Manuscript submitted January 3, 2020, accepted January 17, 2020

${ }^{a}$ Department of Medicine, Jersey Shore University Medical Center, Hackensack Meridian Health, Neptune, NJ 07753, USA

${ }^{\mathrm{b}}$ Corresponding Author: Mujtaba Mohamed, Department of Medicine, Internal Medicine Residency Program, Jersey Shore University Medical Center, Hackensack Meridian Health, Neptune, NJ 07753, USA.

Email: Mujtaba.Mohamed@hackensackmeridian.org

doi: https://doi.org/10.14740/gr1254 gestion and pre-existing hepatic dysfunction. No obvious underlying mechanism to this presentation has been fully explained. Acute renal failure can be associated with this presentation which is even rarer. Stopping IV amiodarone, administering $\mathrm{N}$-acetylcysteine and good supportive care can lead to favorable outcome.

Keywords: Amiodarone; Drug toxicity; Hepatic failure; Renal failure

\section{Introduction}

Amiodarone is a class 3 antiarrhythmic with multiple mechanisms of action, including blocking delayed rectifier potassium channels, calcium channels, and beta adrenergic activity [1]. Its ability to cause hepatotoxicity with chronic use has been thoroughly described in literature. However, evidence has emerged that implicates the use of intravenous (IV) amiodarone with acute and fatal hepatotoxicity. The mechanism behind this acute toxicity has not been fully elucidated, but has been hypothesized to involve free radical formation in the setting of poor liver perfusion, resulting in hypoxic damage to the hepatocytes [2]. It is a fatal complication and associated with a high mortality. Herein, we are presenting a case of acute liver failure and renal failure induced by a single dose of IV amiodarone.

\section{Case Report}

A 67-year-old man with past medical history of coronary artery disease (CAD) status post coronary artery bypass graft (CABG), history of alcoholism, and history of chronic kidney disease stage 3 presented with chest pain for 1 week. Chest pain was intermittent, left sided, 8/10 in severity, non-radiating, dull in nature, exacerbated by exertion and mildly relieved by rest. Remaining review of system was negative. He stopped taking all his cardiac medications for 1 year. Vitals were as follows: blood pressure 130/76 mm Hg, pulse 142/beat per minute respiratory rate $18 / \mathrm{min}$. Pulse oximetry was $97 \%$ on $2 \mathrm{~L}$ nasal cannula. Cardiac examination was significant for tachycardia with irregularly irregular pulse but no murmur, rub or gallops. Lungs were clear to auscultation. Other system examinations were unremarkable. Laboratory data showed sodium 137 (136 


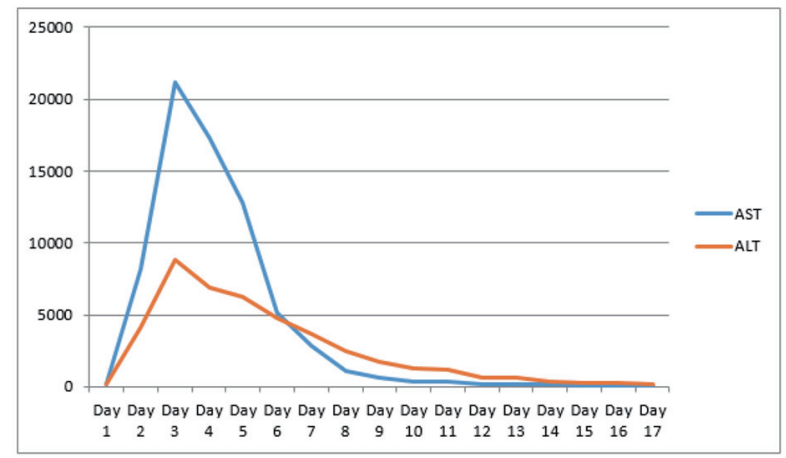

Figure 1. AST and ALT levels through hospital stay. AST: aspartate transaminase; ALT: alanine transaminase.

- $145 \mathrm{mmol} / \mathrm{dL})$, potassium $5.1(3.5-5.2 \mathrm{mmol} / \mathrm{dL})$, chloride $105(96-110 \mathrm{mmol} / \mathrm{dL})$, bicarbonate $22(24-31 \mathrm{mmol} / \mathrm{dL})$, blood urea nitrogen (BUN) $37(5-25 \mathrm{mg} / \mathrm{dL})$, creatinine 1.85 $(0.44-1.0 \mathrm{mg} / \mathrm{dL})$, glucose $130(70-99 \mathrm{mg} / \mathrm{dL})$, aspartate transaminase (AST) $176(10-42 \mathrm{IU} / \mathrm{L})$, alanine transaminase (ALT) 208 (10 - $60 \mathrm{IU} / \mathrm{L})$, international normalized ratio (INR) 1.39, and thyroid stimulating hormone (TSH) 6.87 (0.3 - 4.5 $\mathrm{IU} / \mathrm{mL}$ ). Serum troponin was 0.01 (reference value $<0.04 \mathrm{ng} /$ $\mathrm{mL}$ ). Electrocardiogram (EKG) showed 2:1 atrial flutter. Initially patient was given cardizem and $\beta$-blocker boluses but heart rate did not improve. He was loaded with amiodarone $150 \mathrm{mg}$ intravenous push (IVP) over $10 \mathrm{~min}$ and continued on amiodarone drip $1 \mathrm{mg} / \mathrm{min}$ over $6 \mathrm{~h}$. Heparin drip was started for anticoagulation. On the following day morning labs showed significantly elevated liver enzymes and worsening renal function from his base line on the day of admission. AST was 4,250 $(10$ - $42 \mathrm{IU} / \mathrm{L})$, ALT 2,422 (10 - $60 \mathrm{IU} / \mathrm{L})$, and INR 2.28. Creatinine was $3.2 \mathrm{mg} / \mathrm{dL}(0.44-1.0 \mathrm{mg} / \mathrm{dL})$, and BUN $44 \mathrm{mg} / \mathrm{d}$ reference value $(5-25 \mathrm{mg} / \mathrm{dL})$. At that moment patient was hemodynamically stable with blood pressure (BP) of 105/55 $\mathrm{mm} \mathrm{Hg}$, HR 70 beats per min and pulse ox of $95 \%$ on room air. Six hours later, patient became confused and unable to protect his airways. A rapid response was called, and the patient was intubated for airway protection and transferred to intensive care unit (ICU). Amiodarone was immediately stopped. Right upper quadrant ultrasound, a computed tomography (CT) of the chest, abdomen and pelvis without contrast were normal. Blood cultures, toxicology screen including acetaminophen level, hepa-

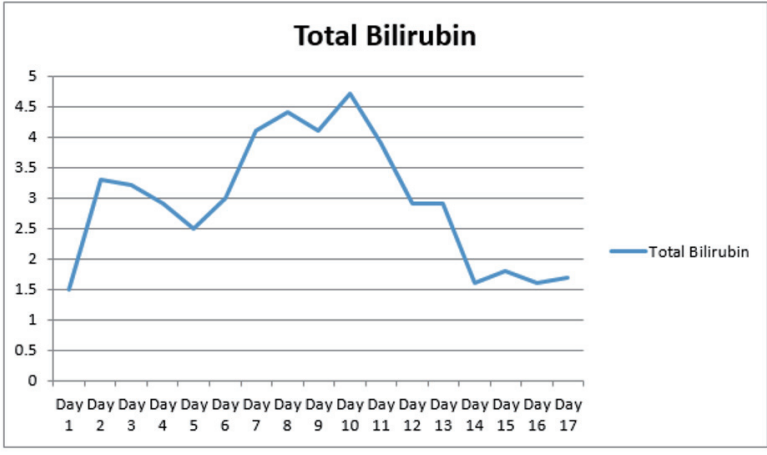

Figure 2. The trend of total bilirubin during the hospital stay.

titis panel, antinuclear antibodies (ANAs), anti-smooth muscle antibody (ASMA), antimitochondrial antibody (AMA) were all negative. Alpha-1 antitrypsine (A1AT) was $126(90-200 \mathrm{mg} /$ $\mathrm{dL})$, and ceruloplasmin $18(17-54 \mathrm{mg} / \mathrm{dL})$. Hereditary hemochromatosis mutation with $\mathrm{C} 282 \mathrm{Y}$ heterozygote and $\mathrm{H} 63 \mathrm{D}$ was negative. Patient was immediately started on $\mathrm{N}$-acetylcysteine drip for $72 \mathrm{~h}$. Patient's liver enzymes continued to rise and peaked at 21,000 (10 - 42 IU/L) for AST and 9,020 (10 - 60 IU/L) for ALT (Fig. 1, Table 1). Total bilirubin increased to 3.9 (Fig. 2, Table 1). INR was 3.8 (day 3 of admission) (Fig. 3). Patient was deemed not a candidate for liver transplant due to history of alcoholism and acute-on-chronic kidney injury. As patient's kidney function continued to worsen, he was started on hemodialysis (on the fifth day). His platelets count began to drop on day 3. Heparin-induced thrombocytopenia was suspected and heparin was stopped. Heparin-induced platelet antibodies with reflex to serotonin were positive. Patient was started on argatroban but serotonin release assay came back negative. Patient was successfully extubated to nasal cannula on day 8. LFTs improved and came down AST 79, ALT 192, and albumin of 2.5. INR was falsely high when patient was receiving argatroban (Fig. 3) but improved after argatroban was stopped. Kidney function eventually improved, and he did not need more hemodialysis.

\section{Discussion}

Amiodarone is an iodinated-benzofuran class 3 antiarrhyth-

Table 1. Summary of Laboratory Investigations Baseline After Amiodarone and Follow-Up After Stopping Amiodarone

\begin{tabular}{llllll}
\hline Biochemistry & Baseline & $\mathbf{2 6} \mathbf{h}$ after amiodarone & At $\mathbf{7 2} \mathbf{h}$ & At $\mathbf{1 8}$ day follow-up & Reference values \\
\hline Blood urea nitrogen & 37 & 44 & 53 & 17 & $5-25 \mathrm{mg} / \mathrm{dL}$ \\
Creatinine & 1.85 & 3.25 & 4.3 & 1.3 & $0.61-1.24 \mathrm{mg} / \mathrm{dL}$ \\
Glomerular filtration rate & $>37$ & 19 & 14 & $>54$ & $70-99 \mathrm{mg} / \mathrm{dL}$ \\
AST & 176 & 4,250 & 21,700 & 79 & $10-42 \mathrm{IU} / \mathrm{L}$ \\
ALT & 208 & 2,422 & 9,020 & 192 & $10-60 \mathrm{IU} / \mathrm{L}$ \\
Alkaline phosphatase & 94 & 84 & 65 & 146 & $38-126 \mathrm{IU} / \mathrm{L}$ \\
Total bilirubin & 1.5 & 3.9 & 2.9 & 1.7 & $0.2-1.3 \mathrm{mg} / \mathrm{dL}$ \\
\hline
\end{tabular}

AST: aspartate aminotransferase; ALT: alanine transaminase. 


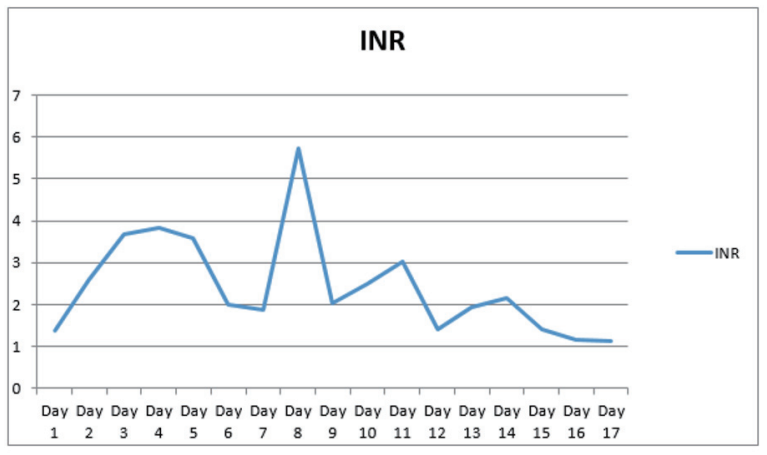

Figure 3. INR trend during hospital stay. INR: international normalized ratio.

mic medication. It is very effective against a wide group of ventricular tachyarrhythmias. It can be administered via oral or IV route. It is an extremely lipophilic medication which facilitates its penetration to various tissues in the body including liver tissue. It has long half-life (6 months); therefore, most of the side effects are associated with long-term use. Side effects associated with prolonged use of amiodarone are hypothyroidism, hyperthyroidism, lung fibrosis, liver toxicity, QT prolongation, and hypotension. Hepatotoxicity caused by oral amiodarone is well documented with around $15-20 \%$. In contrast to long-term side effects, acute side effects of amiodarone associated with IV route are rare but more deleterious. Acute side effects include acute liver failure, cardiac arrest, acute respiratory distress syndrome, renal injury, and hypotension, which are the most common [3-8]. Acute hepatic failure due to IV amiodarone is extremely rare with an incidence of $3 \%$ [9]. The underlying mechanism is still not fully understood. However, a case-control retrospective study argues that hepatotoxicity due to IV amiodarone cannot be differentiated from ischemic hepatitis from concomitant hypotension and claims that they are in fact the same entity [10]. IV amiodarone is formulated with polysorbate 80 as a solubilizer, which has been shown to induce hypotension and increase the systemic exposure of several medications [11]. In this case, patient had a stable blood pressure when amiodarone was started and even when his LFTs increased on the following morning. IV amiodarone toxicity can raise AST/ALT up to 100 - 200 folds [7] (AST of 21,000 and ALT of 9,020 in our case) within a day of infusion which then reverses quickly after discontinuation of amiodarone. In our case, liver function tests (LFTs) increased after $26 \mathrm{~h}$ of starting IV amiodarone and peaked after $72 \mathrm{~h}$ of stopping the infusion (Table 1, Fig. 1). Based on a case review, all cases of acute liver failure due to amiodarone did not survive except for one case [12]. The most common cause of death is encephalopathy [12]. In the case that survived, $\mathrm{N}$-acetylcysteine was administered. Our patient received the same treatment. The mechanism of $\mathrm{N}$-acetylcysteine in nonacetaminophen intoxication patient is unclear. However, some theories suggest that $\mathrm{N}$-acetylcysteine may benefit such patients by improving systemic hemodynamics, tissue oxygen delivery and liver perfusion or through other favorable effects on the acutely injured liver [13]. However, Chughlay et al reported in a systemic review that there was no difference in the primary outcomes of overall survival at 3 weeks between $\mathrm{N}$-acetylcysteine $(70 \%)$ and placebo $(66 \%)$ in non-paracetamol non-paracetamol drug-induced liver injury [14]. According to the same meta-analysis $\mathrm{N}$-acetylcysteine significantly improved the secondary outcomes of transplant-free survival compared with placebo by $40 \%$ versus $27 \%$ for placebo [14]. More studies should be done before recommending this medication in this condition. The fatal dose of IV amiodarone is 1,200 to $1,500 \mathrm{mg}$ [15]. Our patient received $150 \mathrm{mg}$ loading dose of amiodarone, $1 \mathrm{mg} / \mathrm{kg} / \mathrm{min}$ for $6 \mathrm{~h}(360 \mathrm{mg})$ and 0.5 $\mathrm{mg} / \mathrm{kg} / \mathrm{min}$ for $12 \mathrm{~h}(360 \mathrm{mg})$. According to systemic review by Nasser et al, hepatic congestion and low ejection fraction can be a risk factor for acute hepatic failure associated with IV amiodarone [2]. Our patient had an ejection fraction of 40$45 \%$, but liver ultrasound was negative for liver congestion. In addition, our patient had history of alcohol use disorder which can be an additional risk factor. James et al mentioned that oral amiodarone can be safely started once the liver enzymes normalize [16]. However our patient was not continued on oral amiodarone after resolution of acute hepatic failure. Regarding acute renal failure with IV amiodarone there is no much literature on this topic. Reported incidence of increase of creatinine is $8.8 \%$ in patients on oral amiodarone with mean increase of $0.81 \mathrm{mg} / \mathrm{dL}[16,17]$. Paudel et al reported a case of acute renal failure concomitant with acute hepatic failure [18]. However, the patient they reported had hypotension with systolic pressure of 86 during the amiodarone infusion. Our patient developed acute renal failure without hypotension or shock and underwent hemodialysis. Underlying cause of his acute renal failure was possibly due to acute tubular necrosis from amiodarone toxicity. He had two sessions of hemodialysis. Kidney functions improved afterwards.

\section{Conclusions}

Acute hepatic failure secondary to IV amiodarone is extremely rare presentation. It is even rarer to be concomitant with acute renal failure. It usually occurs within first $24 \mathrm{~h}$ of receiving IV amiodarone. Mechanism of this toxicity is not fully understood. Ischemic hepatitis and polysorbate 80-induced hypotension are theories to explain the mechanism. However, our patient never developed hypotension. Clinician should be aware of this potential life-threatening complication and if possible IV amiodarone should be avoided in patient with pre-existing liver disease. Also further research is needed to explain the mechanism and role of $\mathrm{N}$-acetylcysteine in treatment of this condition.

\section{Acknowledgments}

We would like to thank Chunwei, Ma (Liberian at Jersey Shore University Medical Center) for helping providing us with other case reports of intravenous amiodarone-induced acute hepatic failure for comparison with our case. 


\section{Financial Disclosure}

Authors have no financial interest to disclose.

\section{Conflict of Interest}

The authors declare that there is no conflict of interest regarding the publication of this paper.

\section{Informed Consent}

Written informed consent was obtained from the patient for publication of this case report and any accompanying images.

\section{Author Contributions}

$\mathrm{MH}, \mathrm{CK}, \mathrm{AM}$, and AA encouraged MM, AA and MF to learn about acute hepatic failure induced by intravenous amiodarone and how it presents and its management. All authors discussed the medical literature. MM presented the idea; AA and MF wrote the manuscript with input from all authors. Each author has individually been involved and participated in drafting the manuscript and revising it critically for important intellectual content and have given final approval of the version to be published. Each has agreed to be accountable for all aspects of the work in ensuring that questions related to the accuracy or integrity of any part of the work are appropriately investigated and resolved.

\section{Data Availability}

The authors declare that data supporting the findings of this study are available within the article.

\section{References}

1. Smith TW, Cain ME. Class III antiarrhythmic drugs: amiodarone, ibutilide, and sotalol. In: Zipes DP, Jalife J, editors. Cardiac electrophysiology: from cell to bedside. 5th ed. Philadelphia, WB Saunders; 2009: 932-941.

2. Nasser M, Larsen TR, Waanbah B, Sidiqi I, McCullough PA. Hyperacute drug-induced hepatitis with intravenous amiodarone: case report and review of the literature. Drug Healthc Patient Saf. 2013;5:191-198.

3. Lardinois D, Handschin A, Weder W. Acute amiodaroneinduced pulmonary toxicity after lung operation. Ann
Thorac Surg. 2002;73(6):2033-2034; author reply 2034.

4. McGovern B, Garan H, Kelly E, Ruskin JN. Adverse reactions during treatment with amiodarone hydrochloride. Br Med J (Clin Res Ed). 1983;287(6386):175-180.

5. Kumar S, Bangalore S, Kumari R, Grosu H, Jean R. Amiodarone-induced acute respiratory distress syndrome masquerading as acute heart failure. J Emerg Med. 2012;43(5):e311-314.

6. Liverani E, Armuzzi A, Mormile F, Anti M, Gasbarrini G, Gentiloni N. Amiodarone-induced adult respiratory distress syndrome after nonthoracotomy subcutaneous defibrillator implantation. J Intern Med. 2001;249(6):565-566.

7. Fogoros RN, Anderson KP, Winkle RA, Swerdlow CD, Mason JW. Amiodarone: clinical efficacy and toxicity in 96 patients with recurrent, drug-refractory arrhythmias. Circulation. 1983;68(1):88-94.

8. Harris L, McKenna WJ, Rowland E, Holt DW, Storey GC, Krikler DM. Side effects of long-term amiodarone therapy. Circulation. 1983;67(1):45-51.

9. Desai AD, Chun S, Sung RJ. The role of intravenous amiodarone in the management of cardiac arrhythmias. Ann Intern Med. 1997;127(4):294-303.

10. Gluck N, Fried M, Porat R. Acute amiodarone liver toxicity likely due to ischemic hepatitis. Isr Med Assoc J. 2011;13(12):748-752.

11. Schwartzberg LS, Navari RM. Safety of polysorbate 80 in the oncology setting. Adv Ther. 2018;35(6):754-767.

12. Jaiswal P, Attar BM, Yap JE, Devani K, Jaiswal R, Wang Y, Szynkarek R, et al. Acute liver failure with amiodarone infusion: A case report and systematic review. J Clin Pharm Ther. 2018;43(1):129-133.

13. Harrison PM, Wendon JA, Gimson AE, Alexander GJ, Williams R. Improvement by acetylcysteine of hemodynamics and oxygen transport in fulminant hepatic failure. N Engl J Med. 1991;324(26):1852-1857.

14. Chughlay MF, Kramer N, Spearman CW, Werfalli M, Cohen K. N-acetylcysteine for non-paracetamol druginduced liver injury: a systematic review. Br J Clin Pharmacol. 2016;81(6):1021-1029.

15. Kalantzis N, Gabriel P, Mouzas J, Tiniakos D, Tsigas D, Tiniakos G. Acute amiodarone-induced hepatitis. Hepatogastroenterology. 1991;38(1):71-74.

16. James PR, Hardman SM. Acute hepatitis complicating parenteral amiodarone does not preclude subsequent oral therapy. Heart. 1997;77(6):583-584.

17. Chan AL, Hsieh HJ, Hsieh YA, Lin SJ. Fatal amiodarone-induced hepatotoxicity: a case report and literature review. Int J Clin Pharmacol Ther. 2008;46(2):96-101.

18. Paudel R, Dogra P, Suman S, Acharya S, Matta J. Acute liver and renal failure: a rare adverse effect exclusive to intravenous form of amiodarone. Case Rep Crit Care. 2016;2016:5232804. 